\title{
On the light refraction and polarisation in the pres- ence of weak optical activity
}

\author{
Zapeka B., Kostyrko M. and Vlokh R. \\ Institute of Physical Optics, 23 Dragomanov St., 79005 Lviv, Ukraine, \\ e-mail:vlokh@ifo.lviv.ua
}

Received: 30.01.2010

\begin{abstract}
We show that the weak optical activity should manifest itself in changing refractive indices and optical birefringence of a medium. Increments of both the refractive indices and the birefringence which appear due to the weak optical activity are quite small and depend on the signs of neither the optical activity vector nor the light wave vector.
\end{abstract}

Keywords: weak optical activity, gyration, spatial dispersion, refraction, polarisation

PACS: $78.20 . \mathrm{Ek}$

UDC: 535.56

\section{Introduction}

It is well known that the optical activity is described by accounting of inhomogeneity of electric induction $D_{j}$ in relation to electric field $E_{i}$ of an optical wave propagating through a medium:

$$
E_{i}=B_{i j}^{0} D_{j}+i \gamma_{i j k} \frac{\partial D_{j}}{\partial x_{k}}
$$

where $B_{i j}^{0}$ denotes the optical-frequency impermeability tensor, $\gamma_{i j k}$ third-rank antisymmetric polar tensor $\left(\gamma_{i j k}=-\gamma_{j i k}\right)$ and $x_{k}$ the coordinate. Using the known duality relation,

$$
\frac{2 \pi}{\lambda} \gamma_{i j k}=\delta_{i j l} g_{l k}
$$

one can write the tensor $\gamma_{i j k}$ as an axial nonsymmetric second-rank gyration tensor $g_{l k}$ (with $\delta_{i j l}$ being the unit antisymmetric Levi-Civita tensor). Thus, Eq. (1) may be rewritten as

$$
E_{i}=\left(B_{i j}^{0}+i \delta_{i j l} g_{l k} m_{k}\right) D_{j},
$$

where $k_{k}=\frac{2 \pi}{\lambda} m_{k}$ is the wave vector of light and $m_{k}$ the unit vector parallel to $k_{k}$. The nonsymmetric gyration tensor may be decomposed in the symmetric and antisymmetric parts: 


$$
g_{l k}=g_{l k}^{s}+g_{l k}^{a s},
$$

A common point of view is that the optical activity effect is purely associated with the symmetric part of the gyration tensor. Concerning its antisymmetric part which is dual to some polar vector $h_{r}$,

$$
g_{l k}^{a s}=\delta_{l k r} h_{r},
$$

and describes a so-called weak optical activity [1], only a few mentions may be found in the literature. As far as we know, the latter phenomenon has not yet been discovered. However, some data are available in the literature on the observation of this effect by indirect optical techniques, i.e. by changes observed in the exciton reflection and luminescence spectra [2,3]. It has been shown in the work [4] that the weak optical activity can be detected by ellipsometric analysis of the light reflected from relevant gyrotropic crystals. Moreover, it has been stated in [5] that the reflection spectroscopy is the only method enabling any experimental studies of the weak optical activity. Besides, as mentioned in the work [6], this kind of optical activity should cause no changes in the refractive indices of weakly optically active media.

The present work is devoted to description of weak optical activity in crystals and analysis of manifestations of this phenomenon.

\section{Polarisation of optical waves}

Let us consider the weak optical activity in a more detail. Since the vector $h_{r}$ is a constitutive coefficient of a medium, its non-zero value would be peculiar only of polar media. Actually, the point symmetry groups for which $h_{r} \neq 0$ are 1, 2, m, mm2, 3, 3m, 4, 4mm, $6,6 \mathrm{~mm}, \infty$ and $\infty \mathrm{mm}$. Among these groups, only four do not reveal the usual optical activity $\left(g_{l k}^{s}=0\right)$, namely $3 m, 4 m m, 6 m m$ and $\infty m m$. For those media Eq. (3) may be written as

$$
E_{i}=\left(B_{i j}^{0}+i \delta_{i j l} \delta_{l k r}\left[h_{r} \times m_{k}\right]\right) D_{j} .
$$

It is obvious that only a single component of the $h_{r}$ vector remains non-zero for the crystals belonging to the point groups $3 m, 4 m m, 6 m m$, e.g., $h_{3} \neq 0$. Hence, the waves propagating along $\mathrm{Z}$ axis should not be affected by the weak optical activity because the vector product is $\left[h_{3} \times m_{3}\right]=0$ in this case.

Notice that the imaginary part of the optical-frequency impermeability tensor $i \delta_{i j l} \delta_{l k r}\left[h_{r} \times m_{k}\right]$ is antisymmetric either for the usual or weak optical activity effects, being dual to an axial vector. For example, for the propagation of light along $X$ axis we may write

$$
E_{i}=\left(B_{i j}^{0}+i \delta_{i j 2} \delta_{213}\left[h_{3} \times m_{1}\right]\right) D_{j}
$$

where the indices $i, j$ are equal to 1,3 or 3,1 , respectively. Then we have 


$$
\begin{aligned}
& E_{1}=\left(B_{13}^{0}+i \delta_{132} \delta_{213}\left[h_{3} \times m_{1}\right]\right) D_{3} \text { or } \\
& E_{3}=\left(B_{31}^{0}+i \delta_{312} \delta_{213}\left[h_{3} \times m_{1}\right]\right) D_{1}
\end{aligned}
$$

Since $B_{31}^{0}=B_{13}^{0}=0$ and $\delta_{312}=1, \delta_{213}=-1, \delta_{132}=-1$, one can present Eqs. ( 8 ) as

$$
\begin{aligned}
& \Delta E_{1}=E_{1}-B_{13}^{0} D_{3}=\left[i\left[h_{3} \times m_{1}\right] \times D_{3}\right] \text { or } \\
& \Delta E_{3}=E_{3}-B_{31}^{0} D_{1}=\left[-i\left[h_{3} \times m_{1}\right] \times D_{1}\right]
\end{aligned}
$$

where $\left[h_{3} \times m_{1}\right]=\rho_{2}$ is an axial vector describing rotation of the $E_{i}$ vector around $Y$ axis with respect to the $D_{j}$ vector, i.e.

$$
\Delta E_{1}=i\left[\rho_{2} \times D_{3}\right] \text { or } \Delta E_{3}=-i\left[\rho_{2} \times D_{1}\right] .
$$

Thus, the weak optical activity should manifest itself in appearance of a so-called longitudinal component of the electric field of electromagnetic wave and a longitudinal ellipticity of extraordinary wave (see Figure 1).
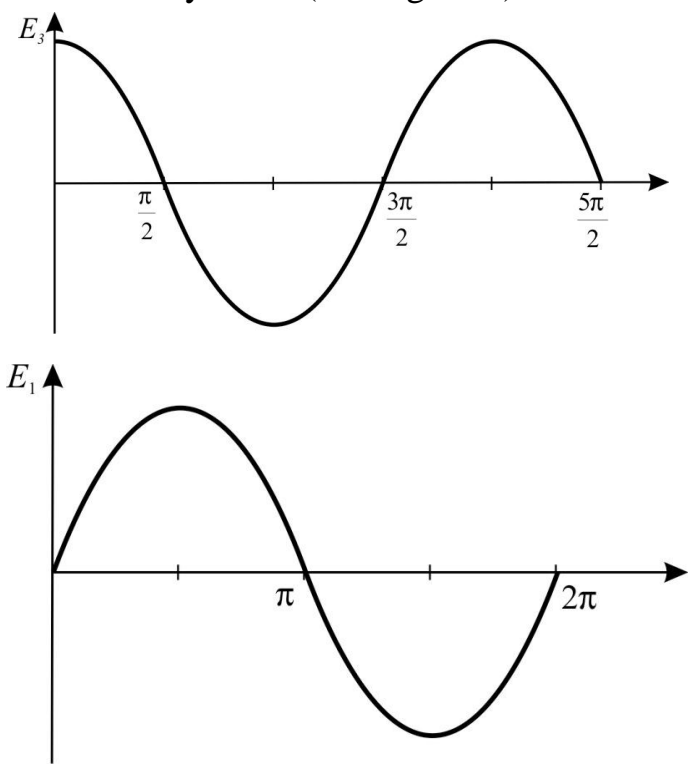

$\mathrm{b}$

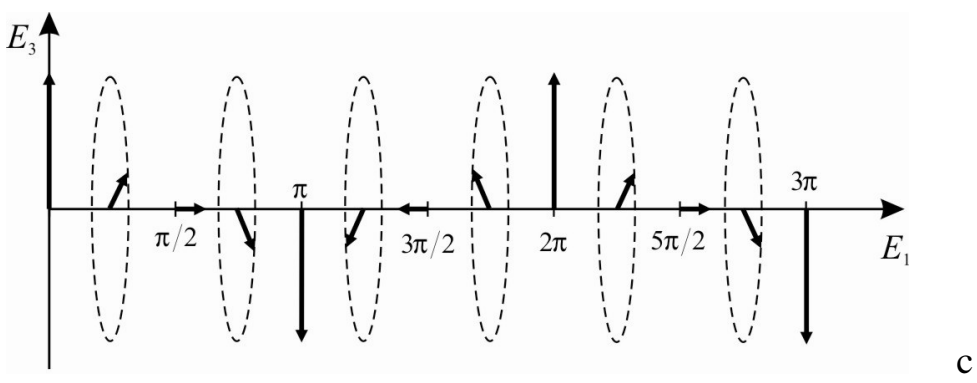

Fig. 1. Schematic representation of electric field components of electromagnetic wave in the presence of weak optical activity $(a, b)$ and ellipticity of one of the eigen waves for this case (c). 
It is seen from the above relations that during each half period the wave becomes purely longitudinal with respect to the wave vector $k_{1}$, though the latter conclusion is not novel (see, e.g., [1,7]). Furthermore, the electromagnetic wave can have only a transverse polarisation due to the well-known relations $(D \bullet k)=0$ and $(E \bullet S)=0$ (with $S$ being the Poynting vector). At this point a number of questions appear, e.g., how the Poynting vector is directed for this longitudinal electric field component of the wave, and whether the weak optical activity influences the refractive indices of the medium?

\section{Refractive indices and the ellipticity of light}

Let us consider the case of optically uniaxial crystals, for which the following conditions are satisfied:

$$
h_{3} \neq 0, h_{1}=h_{2}=0, \quad B_{11}^{0}=B_{22}^{0} \neq B_{33}^{0}, \quad m_{2}=m_{3}=0, \quad m_{1}=1 .
$$

The matrix that couples the components of the electric field and electric displacement vectors may be given by

\begin{tabular}{c|ccc} 
& $D_{1}$ & $D_{2}$ & $D_{3}$ \\
\hline$E_{1}$ & $B_{11}^{0}$ & 0 & $i \rho_{2}$ \\
$E_{2}$ & 0 & $B_{11}^{0}$ & 0 \\
$E_{3}$ & $-i \rho_{2}$ & 0 & $B_{33}^{0}$
\end{tabular},

so that we get a system of equations in the following form:

$$
\left\{\begin{array}{l}
E_{1}=B_{11}^{0} D_{1}+\left[i\left[h_{3} \times m_{1}\right] \times D_{3}\right] \\
E_{2}=B_{11}^{0} D_{2} \\
E_{3}=\left[-i\left[h_{3} \times m_{1}\right] \times D_{1}\right]+B_{33}^{0} D_{3}
\end{array} .\right.
$$

As one can easily see, the refractive index for the wave polarised parallel to the $Y$ axis remains unchanged and is equal to $n_{2}=1 / \sqrt{B_{11}^{0}}$. Accounting for that $E_{1}=D_{1} / n^{2}$ and $E_{3}=D_{3} / n^{2}$, one can reduce the system of Eqs. (13) to that including only two equations:

$$
\left\{\begin{array}{l}
\frac{D_{1}}{n^{2}}=B_{11}^{0} D_{1}+\left[i\left[h_{3} \times m_{1}\right] \times D_{3}\right] \\
\frac{D_{3}}{n^{2}}=\left[-i\left[h_{3} \times m_{1}\right] \times D_{1}\right]+B_{33}^{0} D_{3}
\end{array},\right.
$$

or

$$
\left\{\begin{array}{l}
\left(\frac{1}{n^{2}}-\frac{1}{n_{01}^{2}}\right) D_{1}-\left[i\left[h_{3} \times m_{1}\right] \times D_{3}\right]=0 \\
{\left[i\left[h_{3} \times m_{1}\right] \times D_{1}\right]+\left(\frac{1}{n^{2}}-\frac{1}{n_{03}^{2}}\right) D_{3}=0}
\end{array} .\right.
$$


This system has two positive roots:

$$
n_{3}=n_{03}-\frac{1}{4} \frac{n_{03}^{6}}{\Delta n_{13}}\left[h_{3} \times m_{1}\right]^{2}
$$

and

$$
n_{1}=n_{01}-\frac{1}{4} \frac{n_{01}^{6}}{\Delta n_{13}}\left[h_{3} \times m_{1}\right]^{2}
$$

with

$$
\Delta n_{32}=n_{3}-n_{2}=\Delta n_{32}^{0}-\frac{1}{4} \frac{n_{03}^{6}}{\Delta n_{13}}\left[h_{3} \times m_{1}\right]^{2}
$$

Hence, the ellipticity $\kappa$ of the extraordinary eigen wave is defined by the relation $\frac{D_{1}}{D_{3}}=i \kappa$, and we obtain

$$
\kappa=\frac{\left[h_{3} \times m_{1}\right]^{2}}{\frac{1}{n_{03}^{2}}-\frac{1}{n_{01}^{2}}}=\frac{\bar{n}^{3}\left[h_{3} \times m_{1}\right]^{2}}{2 \Delta n_{13}^{0}} \text { or } \kappa=\frac{\bar{n}^{3} h_{3}^{2}}{2 \Delta n_{13}^{0}}
$$

where $\bar{n}$ is the mean refractive index and $\Delta n_{13}^{0}$ the initial birefringence. The ellipticity of the ordinary eigen wave remains to be zero. The relation for the optical retardation may be represented as

$$
\Delta=\frac{2 \pi d}{\lambda} \Delta n_{32}=\frac{2 \pi d}{\lambda}\left(\Delta n_{32}^{0}-\frac{1}{4} \frac{n_{03}^{6}}{\Delta n_{13}}\left[h_{3} \times m_{1}\right]^{2}\right) .
$$

Here we have taken into account that the two electric field components ( $E_{3}$ and $E_{1}$ ) of the electromagnetic wave appear in the presence of weak optical activity when the incident light propagates along the $X$ direction in crystals or textures belonging to the point symmetry groups $3 \mathrm{~m}, 4 \mathrm{~mm}, 6 \mathrm{~mm}$ and $\infty \mathrm{mm}$. The corresponding components of the Poynting vectors are defined by the relations

$$
S_{1}=\left[E_{3} \times H_{2}\right]
$$

and

$$
S_{3}=\left[E_{1} \times H_{2}\right]
$$

Thus, the electromagnetic energy flow could appear along the $Z$ axis at the cost of reducing the corresponding energy flux along the $X$ axis. This problem will be discussed in our forthcoming papers, along with the problem of parameters of electromagnetic waves in case of coexisting symmetric and antisymmetric parts of the gyration tensor (i.e., both coexisting "strong" and weak optical activities).

\section{Conclusions}

From the relations derived above a number of important conclusions follow. The first is that the weak optical activity should manifest itself in some changes of the refractive in- 
dices and the birefringence. The second is that the increments of the refractive indices and the optical birefringence appearing due to the weak optical activity do not depend on the sign of $h_{3}$ vector, and the same concerns the sign of the wave vector $m_{1}$. Notice that the signs of the rotation of polarisation plane or the phase difference caused by the usual optical activity depend on the wave vector sign.

Finally, the increments of both the refractive indices and the birefringence caused by the weak optical activity are very small, being proportional to the square of the optical activity vector $\left(h_{r}^{2}\right)$. Indeed, assuming that the antisymmetric part of the gyration tensor is of the same order of magnitude as the symmetric one $\left(\sim 10^{-5}\right.$ in common cases), we arrive at the conclusion that the birefringence increment should be of the order of $10^{-7}$. The ellipticity of the eigen extraordinary wave in this case should also be very small $\left(\sim 10^{-7}\right)$.

\section{References}

1. Fedorov F I, 1959. On the theory of optical activity of crystals. 2. Crystals of cubic system and polar classes of middle systems. Opt. Spektrosk. 6: 377-383.

2. Ivchenko E I, Permogorov S A and Selkin A V, 1978. Reflection of light with a change of polarization state from the real crystal boundary. Sol. St. Commun. 28: 345348.

3. Ivchenko E L, Pevtsov A B and Sel'kin A V, 1981. Effects of optical activity on exciton luminescence of CdS. Sol. St. Commun. 39: 453-455.

4. Zil'bershtein A Kh and Solov'ev LE, 1998. Reflection of light with a change of polarization state from real crystal boundary. Opt. Spectr. 84: 549-552.

5. Lalov I J and Kojouharova N A, 2000. Reflection of electromagnetic waves at the boundary of optically active media. Bulgarian J. Phys. 27: 66-71.

6. Agranovich V M and Ginzburg V L. Crystal optics with spatial dispersion, and excitons ( $2^{\text {nd }}$ edition). Moscow: Nauka (1979).

7. Sirotin Yu I and Shaskolskaya M P. Fundamentals of crystal physics. Moscow: Nauka (1979).

Zapeka B., Kostyrko M. and Vlokh R., 2010. On the light refraction and polarisation in the presence of weak optical activity. Ukr.J.Phys.Opt. 11: 68-73.

Анотація. Показано, щзо слабка оптична активність повинна виявлятись у змінах показників заломлення $i$ двозаломлення середовища. Прирости показників заломлення $i$ двозаломлення, щчо з'являються внаслідок слабкої оптичної активності, досить малі $i$ не залежать ні від знака вектора оптичної активності, ні від знака хвильового вектора світла.

Ukr. J. Phys. Opt. 2010, V11, №1 